\title{
Commuting Pattern with Park-and-Ride Option for Heterogeneous Commuters
}

\author{
Chengjuan Zhu, ${ }^{1,2}$ Bin Jia, ${ }^{1,2}$ Linghui Han, ${ }^{3}$ and Ziyou Gao ${ }^{3}$ \\ ${ }^{1}$ State Key Laboratory of Rail Traffic Control and Safety, Beijing Jiaotong University, Beijing 100044, China \\ ${ }^{2}$ School of Traffic and Transportation, Beijing Jiaotong University, Beijing 100044, China \\ ${ }^{3}$ MOE Key Laboratory for Urban Transportation Complex Systems Theory and Technology, Beijing Jiaotong University, \\ Beijing 100044, China
}

Correspondence should be addressed to Bin Jia; bjia@bjtu.edu.cn

Received 8 February 2013; Revised 17 March 2013; Accepted 17 March 2013

Academic Editor: Leman Akoglu

Copyright (C) 2013 Chengjuan Zhu et al. This is an open access article distributed under the Creative Commons Attribution License, which permits unrestricted use, distribution, and reproduction in any medium, provided the original work is properly cited.

\begin{abstract}
We study the effect of the parking on heterogeneous commuters' travel choice in a competitive transportation system which consists of a subway and a parallel road with a bottleneck of limited service capacity. Every morning, commuters either use their private cars only or drive their cars to the bottleneck, park there, and then take the subway to the destination. Considering the effects caused by body congestion in carriage and the parking fees, we developed a bottleneck model to describe the commuters' travel choice. There exist several types of equilibrium that corresponds to user equilibrium. We investigated the influence of the capacity of the bottleneck and the total travel demand on the travel behaviors and on the total social cost. It is shown that there exists a scheme with suitable subway fare and parking fees to implement the minimum total social cost.
\end{abstract}

\section{Introduction}

With the rapid growth of population and the development of urbanization, many researchers focus on the sustainability of transportation operations and pay much attention to the research of more sustainable transportation alternatives such as mass transit in recent years. One of the options is to practice the park-and-ride $(\mathrm{P} \& \mathrm{R})$ systems, in which some travelers drive to a transit station or the parking sites near transit station, and then they park their vehicles and ride in transit to their destinations. P\&R systems are very suitable for the commuting travel from suburban to metropolitan areas because the autoportion of the trip provides connectivity to the P\&R site, while the transit portion enables the transportation of the users to their destinations at a minimal social cost [1]. There exist a number of studies on P\&R systems, including some pieces of research on the policy and design guidelines [2-5] and P\&R location based on computational techniques $[4,6]$. There are also some theoretical analyses. For example, Wang et al. [7] investigated optimal location and pricing of a P\&R facility in a linear city. They presented the necessary conditions for travelers' choice of each mode and formulations to determine optimal parking charges at the P\&R locations. Liu et al. [8] developed a competitive railway/highway system with $\mathrm{P} \& \mathrm{R}$ service in a corridor in which commuters choose between the drive only alternative and the P\&Rs located continuously along the corridor to characterize the equilibrium mode choice. Holguín-Veras et al. [1] studied user rationality and optimal park-andride location under potential demand maximization. These studies did not consider the corridor with a bottleneck constrained. This paper focuses on the influence of parking fee of a P\&R system on the travelers' behavior and the social travel cost in a bottleneck model.

The bottleneck model was proposed by Vickrey [9] and subsequently was refined by Hendrickson and Kocur [10], Daganzo [11], Yang and Huang [12], and so on. In these analyses, commuters must choose their departure time to minimize their travel cost. At user equilibrium, no commuter could reduce their travel cost by unilaterally change his/her departure time. So the commuters' travel cost is decided by his/her departure time choice. Cohen [13] was concerned with two commuter groups: low-income commuters who have lower absolute value of time (VOT) and rigid work 
schedule and high-income commuters with high VOT and flexible work schedule. Arnott et al. [14] considered the welfare effects of congestion tolls with heterogeneous commuters; they treated only cases in which groups differ in one or two parameters. Ramadurai et al. [15] formulated the single bottleneck model with heterogeneous commuters as a linear complementarity problem and proved the existence and uniqueness of the equilibrium solution. Liu and Nie [16] showed two dynamic systems optimal of heterogeneous commuters. Qian et al. [17, 18] investigated how parking locations, capacities, and charges are determined by a private parking market and how they affect the travel patterns and network performances. Zhang et al. [19] integrated the daily commuting patterns, optimal road tolls, and parking fees in a linear city and proposed a time-varying road toll regime to eliminate queuing delay and reduce schedule delay penalty. However, all these studies are limited to the single mode. In a many-to-one network, Zhang et al. [20] introduced parking permits and further verified that parking permits distribution and trading are very efficient in traffic management. Huang [21] compared three pricing schemes in a competitive system with transit and highway for two commuter groups. The body congestion in carriage has no effect on the punctual of the subway but has effect on the crowding discomfort; accordingly, users' traffic behaviors will be changed. Commuters with different social characteristics and different income will have different decision-making behaviors; this is not only present in the departure time choice but also present in the travel mode choice. Huang et al. [22] studied the mode choice and commuting behaviors in a bimodal transportation system with a bottleneck-constrained highway. Van den Berg and Verhoef [23] derived congestion tolling in the bottleneck model with heterogeneous values of time. With a stochastic toll, Yao et al. [24] analyzed the equilibrium departure behavior of heterogeneous risk-averse commuters and formulated and further solved the problem. Xiao et al. [25] considered flat toll and tactical waiting problem under the first-in-first-out (FIFO) queuing discipline.

In congested urban areas, parking of cars is time consuming and sometimes expensive, especially in the center business districts. Urban planners must consider whether and how to accommodate potentially large numbers of cars in the limited geographic areas. Usually the authorities set minimum, or more rarely maximum, numbers of parking spaces for new housing and commercial developments and may also plan its location and distribution to influence its convenience and accessibility. Urban managers usually set reasonable parking fees to regulate the parking market and then to reduce congestion on the ground. The costs or subsidies of such parking accommodations become a heated point in local politics.

We will study a competitive network with bottleneckconstrained highway and P\&R. Parking locations are set at the head and the tail of the bottleneck. Because the bottleneck capacity is limited, commuters must consider the tradeoff among waiting time in the bottleneck queue, in-carriage body congestion, and schedule delay. We begin with a brief review of the travel cost for heterogeneous commuters with different modes in Section 2. We present all possible traffic patterns



FIGURE 1: The simple commuting network.

under the user equilibrium and analyze the traffic behaviors for different parking fees in Section 3. In Section 4, we give the optimal combination of parking fees with minimal total social cost, when both groups use both modes. In Section 5, we focus on the analysis of several equilibrium types and on the optimal parking fees with a minimal total social cost in numerical examples. Finally, in Section 6, the conclusions are shown.

\section{Travel Cost by Car and Park and Ride for Heterogeneous Commuters}

In this section, we describe the problem setup and model assumptions, as well as the notations and definitions used throughout this paper. We assume that, in the morning rush hour, commuters who depart from home $(\mathrm{H})$ at time $t$ drive to their workplace $(\mathrm{W})$ directly or park their car before the bottleneck and then take subway to their office start work at time $t^{*}$, as shown in Figure 1. The bottleneck, with capacity $s$, located at the end of the highway. Queuing usually occurs at the bottleneck when the arrival rate of the cars exceeds its capacity. The capacity constraint is a flow constraint, while the queue discipline is an FIFO.

To keep the analysis manageable, we limit consideration to two groups of commuters, that is, we divide all commuters; into two groups [13] which have different unit costs of travel time $\left(\alpha_{1}\right.$ and $\left.\alpha_{2}\right)$, schedule delay time $\left(\beta_{1}\right.$ and $\beta_{2}$ for early arrival, $\gamma_{1}$ and $\gamma_{2}$ for late arrival), and different unit costs of body congestion in carriage $\left(\theta_{1}\right.$ and $\left.\theta_{2}\right)$. We assume that $\theta_{1}>\theta_{2}, \alpha_{1}>\alpha_{2}, \alpha_{1} / \beta_{1}>\alpha_{2} / \beta_{2}$, and $\gamma_{1} / \beta_{1}=\gamma_{2} / \beta_{2}=\eta$, and that all commuters' work start time is identical. Let $\delta_{i}=$ $\beta_{i} \gamma_{i} /\left(\beta_{i}+\gamma_{i}\right)=\beta_{i} \eta /(1+\eta), i=1,2$. Let $N_{1}$ and $N_{2}$ denote the commuter number of groups 1 and 2 , respectively, and let $N_{1}+N_{2}=N$ hold. Hence, the group with higher unit cost of travel time is more likely to comprise relatively highly paid white-collar workers, with flexible work hours and high VOT; the group with lower unit cost of travel time likely consists of blue-collar worker and clerks, with rigid work schedules and low VOT. The total trip demand is completely inelastic. A car commuter's travel cost consists of the monetary cost of his/her actual travel time, early or late penalty, and the parking fee of the destination. Travel time consists of three aspects, the free-flow travel time, the waiting time at the bottleneck, and the parking time.

It is assumed that without loss of generality that freeflow travel time on road and parking time are zero, so that a commuter by car only reaches the queue at the bottleneck as 
soon as he/she leaves home and arrives at work immediately upon exiting the bottleneck. To simplify, a linear individual travel cost of group $i$ making their trip by car can be expressed as

$$
\begin{aligned}
C_{A i}(t)= & \alpha_{i}(w(t)) \\
& +\max \left\{\beta_{i}\left(t^{*}-t-w(t)\right), \gamma_{i}\left(t+w(t)-t^{*}\right)\right\} \\
& +p_{w}, \quad i=1,2
\end{aligned}
$$

where $w(t)$ denotes the queue waiting time at the bottleneck, $t$ is the departure time from home, and $p_{w}$ is the parking fee of the destination $\mathrm{W}$. We call the cost of arriving at work early $\max \left[0, \beta_{i}\left(t^{*}-t-w(t)\right)\right]$ or late, $\max \left[0, \gamma_{i}\left(t+w(t)-t^{*}\right)\right]$ is the schedule delay cost. Each individual of group 1 decides when to leave home. In doing so, he/she trades off travel time, schedule delay, and the parking fees. Let $N_{A}=N_{A 1}+N_{A 2}$, where $N_{A 1}$ and $N_{A 2}$ denote the number of car mode by group 1 and group 2.

In the combined departure time/parking equilibrium model, all commuters have full information about the traffic conditions and parking fees, and no commuter in each group can unilaterally changing his/her departure time and/or his/her parking location to reduce his/her generalized travel cost at equilibrium. The commuters in each group must have the same commute cost. In this case, group 2 should depart at the center of the rush hour because its relative cost of schedule delay to travel time is higher, whereas group 1 departs on the tails, that is, before or after group 2. Then, the individual travel costs of groups 1 and 2 at equilibrium are

$$
\begin{gathered}
C_{A 1}=\delta_{1} \frac{N_{A 1}+N_{A 2}}{s}+p_{w}, \\
C_{A 2}=\delta_{2} \frac{N_{A 2}}{s}+\frac{\alpha_{2}}{\alpha_{1}} \delta_{1} \frac{N_{A 1}}{s}+p_{w} .
\end{gathered}
$$

The detailed derivation of (2) can be referred to as shown by Arnott et al. [14].

Now, we consider the travel costs incurred on commuters who choose the P\&R. The cost experienced by P\&R commuters should depend on the time spent on the subway more than to drive, the parking fee of $P \& R$, the subway fare, and body congestion in carriages. Then, the total travel cost of a commuter who selects the P\&R mode is

$$
C_{R i}=\alpha_{i} T_{s}+\theta_{i} g\left(N_{R}\right)+P+p_{r}, \quad i=1,2,
$$

where $T_{s}$ is the more time spent on the subway than to drive, $P$ is the subway fare, $p_{r}$ is the parking fee of the P\&R mode, and $g\left(N_{R}\right)$ represents the crowding discomfort generated by body congestion in carriages, $N_{R}=N_{R 1}+N_{R 2}$. Let $g\left(N_{R}\right)=$ $N_{R 1}+N_{R 2}$. So, we have

$$
\begin{aligned}
& N_{A 1}+N_{R 1}=N_{1}, \\
& N_{A 2}+N_{R 2}=N_{2} .
\end{aligned}
$$

\section{User Equilibrium Traffic Profiles}

In this section, we briefly analyze all possible traffic patterns under the user equilibrium for any given set of parking fees and subway fare (i.e., $p_{w}, p_{r}$, and $P$ ). These traffic patterns are central to obtaining the competitive parking equilibrium. We assume $\beta_{1}<\beta_{2}$ to ensure $\alpha_{1} / \beta_{1}>\alpha_{2} / \beta_{2}$, and thus, $\delta_{1}<\delta_{2}$. We also assume that the two groups are not sensitive to the discomfort in the carriage and $\alpha_{1} / \theta_{1}>\alpha_{2} / \theta_{2}$. Nine types of parking lot preference are identified, and they are described as follows.

(1) Both groups only select $P \& R$ mode, which can be expressed as $C_{A 1}>C_{R 1}$ and $C_{A 2}>C_{R 2}$. In this case, $p_{w}-$ $\left(P+p_{r}\right)>\theta_{1} N+\alpha_{1} T_{s}$, the parking fee of the destination $p_{w}$ is so high that all commuters choose $P \& R$ to achieve their trip. And the parking lot of the destination is unable to secure a market share. Since the parking operators in the destination will never have any commuter, under such a parking market, they can always attract commuters by reducing their parking charge.

(2) Both groups select both modes, which can be expressed as $C_{A 1}=C_{R 1}$ and $C_{A 2}=C_{R 2}$, for $N_{i j}>0, i=A, R$; $j=1,2$; that is,

$$
\begin{gathered}
\delta_{1} \frac{N_{A 1}+N_{A 2}}{s}+p_{w}=\alpha_{1} T_{s}+\theta_{1}\left(N_{R 1}+N_{R 2}\right)+P+p_{r} \\
\delta_{2} \frac{N_{A 2}}{s}+\frac{\alpha_{2}}{\alpha_{1}} \delta_{1} \frac{N_{A 1}}{s}+p_{w}=\alpha_{2} T_{s}+\theta_{2}\left(N_{R 1}+N_{R 2}\right)+P+p_{r}
\end{gathered}
$$

for $0<N_{A 1}<N_{1}, 0<N_{R 1}<N_{1}$ and $0<N_{A 2}<N_{2}$, $0<N_{R 2}<N_{2}$.

With the conservation conditions $N_{A 1}+N_{R 1}=N_{1}$ and $N_{A 2}+N_{R 2}=N_{2}$, we have the modal split in equilibrium as follows:

$$
\begin{aligned}
N_{A 1}= & \frac{\delta_{2}+\theta_{2} s}{\delta_{2}-\left(\alpha_{2} / \alpha_{1}\right) \delta_{1}} \\
\times\left[\frac{\left(P+p_{r}-p_{w}\right) s+\alpha_{1} s T_{s}+\theta_{1} s N}{\delta_{1}+\theta_{1} s}\right. & \left.-\frac{\left(P+p_{r}-p_{w}\right) s+\alpha_{2} s T_{s}+\theta_{2} s N}{\delta_{2}+\theta_{2} s}\right], \\
N_{A 2}= & \frac{\left(P+p_{r}-p_{w}\right) s+\alpha_{1} s T_{s}+\theta_{1} s N}{\delta_{1}+\theta_{1} s}-N_{A 1} .
\end{aligned}
$$

Also, we can get $N_{R 1}$ and $N_{R 2}$. The total number of commuters who select car only is

$$
N_{A}=N_{A 1}+N_{A 2}=\frac{\theta_{1} s N+\alpha_{1} s T_{s}+\left(P+p_{r}-p_{w}\right) s}{\delta_{1}+\theta_{1} s},
$$

and the total number of $\mathrm{P} \& \mathrm{R}$ commuters is

$$
N_{R}=N_{R 1}+N_{R 2}=\frac{\delta_{1} N-\alpha_{1} s T_{s}-\left(P+p_{r}-p_{w}\right) s}{\delta_{1}+\theta_{1} s} .
$$

While we are given $T_{s}, P, p_{r}$, and $p_{w},(6)$ shows that the modal split in each group is related to parameters $\theta_{1}$ and $\theta_{2}$. In other ways, (7) and (8) show that the number of P\&R commuters is inversely proportional to $\theta_{1}$, the total usage 
of car or $\mathrm{P} \& \mathrm{R}$ mode depends on the parameter $\theta_{1}$, and the total number of commuters is $N$. Certainly, the equilibrium individual travel costs do not depend on the composition of demand but on the total. While we are given $T_{s}, \theta_{1}$, and $\delta_{1}$, also the capacity of the bottleneck $s$, the total demand, and the modal split only depend on $P+p_{r}-p_{w}$.

The equilibrium occurs when no commuter in one group can reduce his/her travel costs by altering his/her departure time and his/her parking lot. The equilibrium exists only when the values of parameters are in certain ranges and occurs at an interior solution.

(3) Group 1 only selects $P \& R$, while group 2 selects both modes, which can be expressed as $C_{A 1}>C_{R 1}$ and $C_{A 2}=C_{R 2}$, and we can get $N_{A 1}=0, N_{R 1}=N_{1}$, and $\left(\delta_{1}-\delta_{2}\right)\left(P+p_{r}-p_{w}\right)+$ $\left(N_{1}+N_{R 2}\right)\left(\theta_{2} \delta_{1}-\theta_{1} \delta_{2}\right)+\left(\alpha_{2} \delta_{1}-\alpha_{1} \delta_{2}\right) T_{s}>0$. In this case, the inequality can hold with $\delta_{1}<\delta_{2}$ and $P+p_{r}-p_{w}<0$, then the modal split will occur with high enough parking fee of the destination.

(4) Group 2 selects P\&R only, while group 1 selects both modes, which can be expressed as $C_{A 1}=C_{R 1}$ and $C_{A 2}>C_{R 2}$, and then we can get $N_{A 2}=0, N_{R 1}=N_{1}-N_{A 1}$, and $N_{R 2}=$ $N_{2}$. We also have

$$
\begin{gathered}
P+p_{r}-p_{w}<\frac{\alpha_{2} \theta_{1}-\alpha_{1} \theta_{2}}{\alpha_{1}-\alpha_{2}}\left(N_{R 1}+N_{2}\right), \quad N_{R 1}>0, \\
N_{A 1}=N_{A}=\frac{\left(P+p_{r}-p_{w}\right) s+\alpha_{1} s T_{s}+\theta_{1} s N}{\theta_{1} s+\delta_{1}} .
\end{gathered}
$$

Inequality (9) may hold when $\alpha_{1} \theta_{2}>\alpha_{2} \theta_{1},\left(P+p_{r}\right)-p_{w}$ and $N_{2}$ are sufficiently small.

The equilibrium occurs at a corner solution, and group 2 chooses exclusively P\&R mode. Commuters of group 2 only have departure time choice, while commuters of group 1 have both departure time choice and parking lot choice.

(5) Both groups only select car mode, which can be expressed as $C_{A 1}<C_{R 1}$ and $C_{A 2}<C_{R 2}$, then we can get $P+$ $p_{r}-p_{w}>\left(\delta_{1} / s\right) N-\alpha_{2} T_{s}$. Similar to the type (1) equilibrium, all commuters will choose car mode when $p_{w}$ is relatively small. The parking lot of the P\&R is unable to secure a market share. They will reduce their parking fee to attract commuters.

(6) Group 1 only selects car, while group 2 selects both modes, which can be expressed as $C_{A 1}<C_{R 1}$ and $C_{A 2}=C_{R 2}$, and we can get $N_{R 1}=0, N_{A 1}=N_{1}$, and $N_{R 2}=N_{R}=N_{2}-$ $N_{A 2}$. Meanwhile, we have

$$
\begin{gathered}
\left(\alpha_{1}-\alpha_{2}\right)\left(P+p_{r}-p_{w}\right) \\
<\left(\alpha_{2} \theta_{1}-\alpha_{1} \theta_{2}\right) N_{R}+\left(\alpha_{1} \delta_{2}-\alpha_{2} \delta_{1}\right) \frac{N_{A 2}}{s}, \\
N_{A 2}>0 \\
N_{A 2}=\frac{\theta_{2} N_{2} s+\alpha_{2} s T_{s}+\left(P+p_{r}-p_{w}\right) s-\left(\alpha_{2} / \alpha_{1}\right) \delta_{1} N_{1}}{\theta_{2} s+\delta_{2}} .
\end{gathered}
$$

Clearly, inequality (11) may hold when $\alpha_{2} \theta_{1}<\alpha_{1} \theta_{2}, P+$ $p_{r}-p_{w}$ is sufficiently small, and $N_{2}\left(N_{R}\right)$ is sufficiently large. Similarly, the equilibrium occurs at a corner solution, and group 1 chooses exclusively car mode. Commuters of group 1 only have departure time choice, while commuters of group 2 have both departure time choice and parking lot choice.

(7) Group 2 only selects car mode, while group 1 selects both modes, which can be expressed as $C_{A 1}=C_{R 1}$ and $C_{A 2}<$ $C_{R 2}$, and then we can get $N_{R 2}=0$,

$$
\begin{array}{r}
\left(\alpha_{1}-\alpha_{2}\right)\left(P+p_{r}-p_{w}\right) \\
>\left(\alpha_{2} \theta_{1}-\alpha_{1} \theta_{2}\right) N_{R}+\left(\alpha_{1} \delta_{2}-\alpha_{2} \delta_{1}\right) \frac{N_{2}}{s}, \\
N_{A 2}>0 .
\end{array}
$$

The inequality cannot hold when $P+p_{r}-p_{w}<0$; the modal split will not occur.

(8) Group 1 only selects car mode, while group 2 only selects P\&R mode, which can be expressed as $C_{A 1}<C_{R 1}$ and $C_{A 2}>C_{R 2}$, and then we can get $N_{R 1}=0$ and $N_{A 2}=0$; we have $N_{A 1}=N_{1}, N_{R 2}=N_{2}$, and

$$
\begin{gathered}
\frac{\delta_{1}}{s} N_{1}<\alpha_{1} T_{s}+\theta_{1} N_{2}+\left(P+p_{r}-p_{w}\right), \\
\frac{\alpha_{2}}{\alpha_{1}} \frac{\delta_{1}}{s} N_{1}>\alpha_{2} T_{s}+\theta_{2} N_{2}+\left(P+p_{r}-p_{w}\right) .
\end{gathered}
$$

Inequality (14) can hold only when the $P+p_{r}-p_{w}$ is sufficiently small and $N_{2}$ is sufficiently small. Commuters of group 1 only have departure time choice, while commuters of group 2 have neither departure time choice nor parking lot choice.

(9) Group 1 only selects P\&R mode, while group 2 only selects car mode, which can be expressed as $C_{A 1}>C_{R 1}$ and $C_{A 2}<C_{R 2}$, and then we can get $N_{A 1}=0$ and $N_{R 2}=0$; also we have $N_{R 1}=N_{1}, N_{A 2}=N_{2}$, and

$$
\begin{aligned}
& \frac{\delta_{1}}{s} N_{2}>\alpha_{1} T_{s}+\theta_{1} N_{1}+\left(P+p_{r}-p_{w}\right), \\
& \frac{\delta_{2}}{s} N_{2}<\alpha_{2} T_{s}+\theta_{2} N_{1}+\left(P+p_{r}-p_{w}\right) .
\end{aligned}
$$

Inequality (15) cannot hold at the same time with $\delta_{1}<\delta_{2}$; the model split will not occur.

There are nine types of equilibrium. Not all of them are stable both in theory and in practice. We examine them one by one. With the great development of the public transport in China, the parking fee of P\&R is evidently lower than the parking fee of the destination. The types (3), (7), and (9) will not occur with $\delta_{1}<\delta_{2}$ and $P+p_{r}-p_{w}<0$. The types (1) and (5) of equilibrium are theoretically stable, and the operators of one parking lot set a reasonable price and build enough spaces such that they can attract all the commuters. But both types of equilibrium sometimes may not be stable in a practical sense. Since one of the parking operators will never have any commuter under such parking market, they can always attract commuters by reducing their parking charge. The types (6) and (8) of equilibrium are stable in theory. Furthermore, the type (6) only exists under the small portion of group 1 , and the subway fare is sufficiently large; the type (8) only exists under 
a narrow range of prices. So, the two types (6) and (8) may not be desired. The types (2) and (4) of equilibrium are stable both in theory and in practice, because their travel preference and profile exist under a broad range of prices. Since the type (2) equilibrium is equitable to the two groups to choose the two modes and is the most likely to occur in practice, we focus on the analysis of this equilibrium in the following numerical examples.

\section{Optimal Combination of Parking Fees}

From the results in Section 3, it is easily found that the nine equilibrium states cannot happen at the same time, and they are determined by the parking fee for the fixed traffic demand $N_{1}, N_{2}$ and the fare of railway. Therefore, it is needed to study the pricing problem of parking for reducing the total social cost and improving the traffic congestion. In these all possible equilibrium states, the second traffic pattern is the most desirable for traffic managers since all traffic modes are used. In the following, we would design the optimal parking fee based on the second equilibrium traffic pattern (both groups select both modes).

The parking fees problem has two levels of decision making: parking fees setting by an operator, the leader, and then selection of the cheapest alternative by commuters the follower. The game for the leader aims to determine parking fees, such that the total social cost is minimized, while the follower is to minimize his/her travel cost. The total social cost defined in this paper is the sum of all costs borne by subway operator and all commuters, but excluding fares and parking fees. The game is most naturally discussed as a bilevel program. When the lower level attains the Nash equilibrium with two groups using both modes, the lower level can be solved as the constraints of the upper level, so the bilevel problem can be formulated as a mathematical programming with linear constraints. It is assumed that the expenses on labor, fuel, electricity, and routine materials by subway operator are included in the subway fare. The minimization model for the problem can be formulated as

$$
\begin{aligned}
\min & \operatorname{TSC}\left(N_{R 1}, N_{A 1}, N_{R 2}, N_{A 2}, p_{r}-p_{w}\right) \\
= & N_{A 1} \frac{\delta_{1}}{s}\left(N_{A 1}+N_{A 2}\right)+N_{A 2}\left(\frac{\delta_{2}}{s} N_{A 2}+\frac{\alpha_{2}}{\alpha_{1}} \frac{\delta_{1}}{s} N_{A 1}\right) \\
& +N_{R 1}\left[\alpha_{1} T_{s}+\theta_{1}\left(N_{R 1}+N_{R 2}\right)\right] \\
& +N_{R 2}\left[\alpha_{2} T_{s}+\theta_{2}\left(N_{R 1}+N_{R 2}\right)\right]
\end{aligned}
$$

subject to (4)-(5), and all variables are nonnegative. In the objective function of model (16), the first two terms are the total social cost of the car mode commuters, and the last two terms are the total social cost of subway.

The objective function can be simplified as

$$
\begin{aligned}
\text { TSC }= & \left(\alpha_{1} N_{1}+\alpha_{2} N_{2}\right) T_{s} \\
& +\left[\theta_{1} N_{1}+\theta_{2} N_{2}-\left(P+p_{r}-p_{w}\right)\right] N_{R} \\
& +N\left(P+p_{r}-p_{w}\right) .
\end{aligned}
$$

Pluging (8) and $N_{1}+N_{2}=N$ into formula (17), we can get the objective function that can be considered as the function of $P+p_{r}-p_{w}$. So, one of the optimal conditions of the model (16) is

$$
P+p_{r}-p_{w}=-\frac{\alpha_{1} T_{s}+\left(\theta_{1}-\theta_{2}\right) N_{2}}{2} .
$$

The solution of the model is

$$
\begin{aligned}
N_{A}= & \frac{2 \theta_{1} N_{1} s+\alpha_{1} T_{s} s+\left(\theta_{1}+\theta_{2}\right) N_{2} s}{2\left(\delta_{1}+\theta_{1} s\right)}, \\
N_{R}= & \frac{2 \delta_{1} N-\alpha_{1} s T_{s}+\left(\theta_{1}-\theta_{2}\right) N_{2} s}{2\left(\delta_{1}+\theta_{1} s\right)}, \\
N_{A 2}= & \left(\alpha_{2} T_{s}+\left(\theta_{2}+\frac{\alpha_{2}}{\alpha_{1}} \frac{\delta_{1}}{s}\right) N_{R}\right. \\
& \left.-\frac{\alpha_{2}}{\alpha_{1}} N-\frac{\alpha_{1} T_{s}+\left(\theta_{1}-\theta_{2}\right) N_{2}}{2}\right) \\
& \times\left(\frac{\delta_{2}}{s}-\frac{\alpha_{2}}{\alpha_{1}} \frac{\delta_{1}}{s}\right)^{-1},
\end{aligned}
$$

and other variables $N_{R 1}, N_{A 1}$, and $N_{R 2}$ can be computed by (18)-(19).

The optimal total social cost of the other types of equilibrium can be computed in the same way, and one only needs to substitute the constraints by the corresponding formulations. The results show that the variation of the parking fee of $P \& R$ only influences the travel cost of commuters and the optimal parking fare of the destination, but it has no effect on the flow distribution and the total social cost.

\section{Numerical Examples}

Now we give numerical examples to support our analyses and to illustrate some insights into the characteristics of the flexible parking fees in the long term. The basic model parameters are as follows: the unit costs of body congestion of group 2 are $\theta_{2}=0.10$ (Yuan/discomfort equivalent), $\left(\alpha_{1}, \beta_{1}, \gamma_{1}\right)=$ $(1.2,0.5,1.5)$ (Yuan/min), $\left(\alpha_{2}, \beta_{2}, \gamma_{2}\right)=(0.8,0.6,1.8)$ (Yuan/ $\min$ ), and subway fare $P=2$ (Yuan). Allow the total number of commuters to change from 200 to 300 , and keep the relative shares of the two groups unchanged, 0.5 .

5.1. Case 1. Set the unit costs of body congestion of group 1 $\theta_{1}=0.105$. Let the capacity of the bottleneck change from 2 to 4 and $T_{s}$ change from 4 to 6 .

When the total demand is set to be $N=250$, the modal splits and the total social cost influenced by $s$ and $T_{s}$ are shown in Figures 2, 3, and 4.

In Figures 2 and 3, it is found that both the car usage in group 1 and the total car usage increase with the capacity of the bottleneck and $T_{s}$. Both Figures 2 and 3 illustrate that a higher capacity of the bottleneck and $T_{s}$ attract more car commuters, especially more car commuters in group 1 , which is consistent with the fact. When $s=4$, the car commuters in group 1 decreases sharply then slowly with the decrease of the $T_{s}$, but the total number of car commuters decreases slowly. 


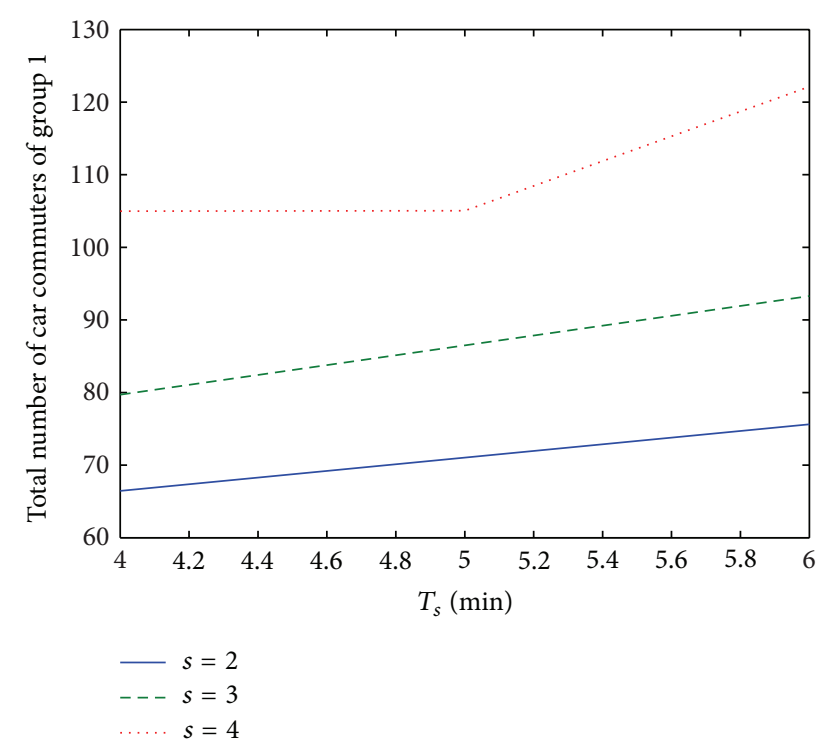

Figure 2: $N_{A 1}$ versus $T_{s}$ with different $s$.



Figure 3: $N_{A}$ versus $T_{s}$ with different $s$.

Figure 4 displays the total social cost with different $s$ and $T_{s}$. It shows that, on the one hand, as the capacity of the bottleneck increases, the total social cost becomes smaller; on the other hand, as the time $T_{s}$ becomes bigger, the total social cost becomes larger. It can be seen that through the implementation of traffic management to improve the capacity of the bottleneck or reduce the time spent on the subway one can cut down the total social cost to some extent.

When the capacity of the bottleneck is set to be $s=3$, the modal splits and the total social cost influenced by $T_{s}$ with different total demand are shown in Figures 5, 6, and 7.



Figure 4: Total social cost versus $T_{s}$ with different $s$.

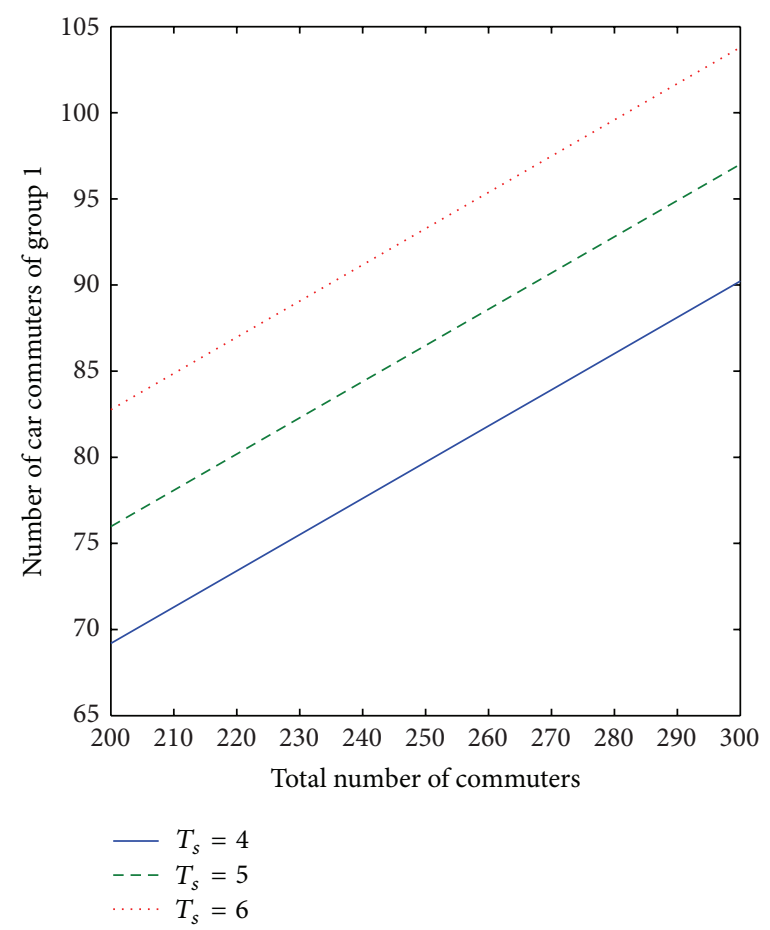

FIgURe 5: $N_{A 1}$ versus $N$ with different $T_{s}$.

Figures 5 and 6 depict the number of car commuters of group 1 and total number of car commuters with different total demand for different $T_{s}$. Both the car usage in group 1 and the total car usage increase linearly with the total demand. This reflects the fact that the less time on subway, the less people in cars. As $T_{s}$ becomes smaller, the impact on the total car usage becomes less marked due to the opposite travel choice behavior in group 2. Moreover, the impact on the total social cost is also insignificant, as shown in Figure 7. The total social cost increases with the total demand and $T_{s}$ 




Figure 6: $N_{A}$ versus $N$ with different $T_{s}$.



FIgURE 7: Total social cost versus $N$ with different $T_{s}$.

at a certain demand level. This is because the increase of the total demand induced higher total time cost, queuing delay cost, and congestion cost in carriage.

5.2. Case 2. Set the bottleneck capacity $s=3$ (veh/min), $T_{s}=$ 4 ( $\mathrm{min}$ ), and $p_{r}=2$ (yuan). Let the unit costs of body congestion of group 1 vary from 0.105 to 0.12 . In Figures 8 and 9 , the number of the car commuters in group 1 and total car commuters with different demand and $\theta_{1}$ are shown. As the service level of the subway improved, $\theta_{1}$ becomes lower, and more and more commuters of group 1 give up the direct drive and choose $P \& R$, but the variation on the total car usage is inconspicuous due to the opposite travel choice behavior in group 2 .

When the total demand is set to be $N=250$, the total social cost influenced by the unit costs of body congestion of group 1 is shown in Figure 10. It shows that the total social cost increases sharply first with the service level of the subway

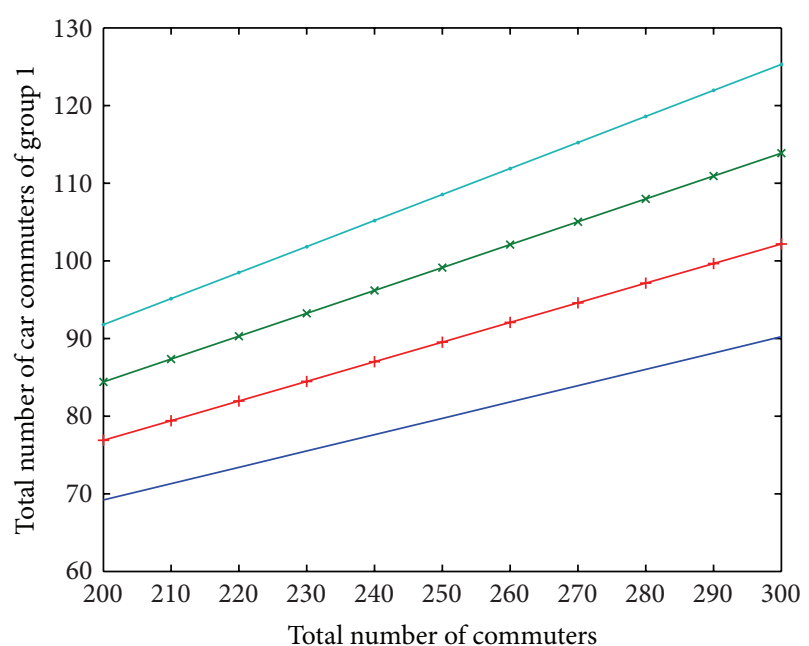

$-\theta_{1}=0.105 \quad+\theta_{1}=0.115$

$\rightarrow \theta_{1}=0.110 \longrightarrow \theta_{1}=0.120$

FIgURE 8: $N_{A 1}$ versus total demand with different $\theta_{1}$.

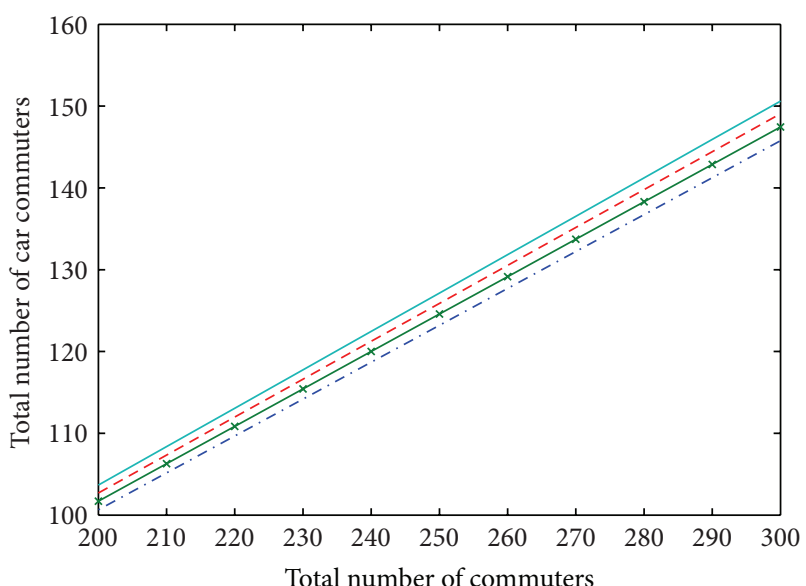

$\cdots-\theta_{1}=0.105 \quad--\theta_{1}=0.115$

$\rightarrow \theta_{1}=0.110-\theta_{1}=0.120$

Figure 9: $N_{A}$ versus total demand with different $\theta_{1}$.

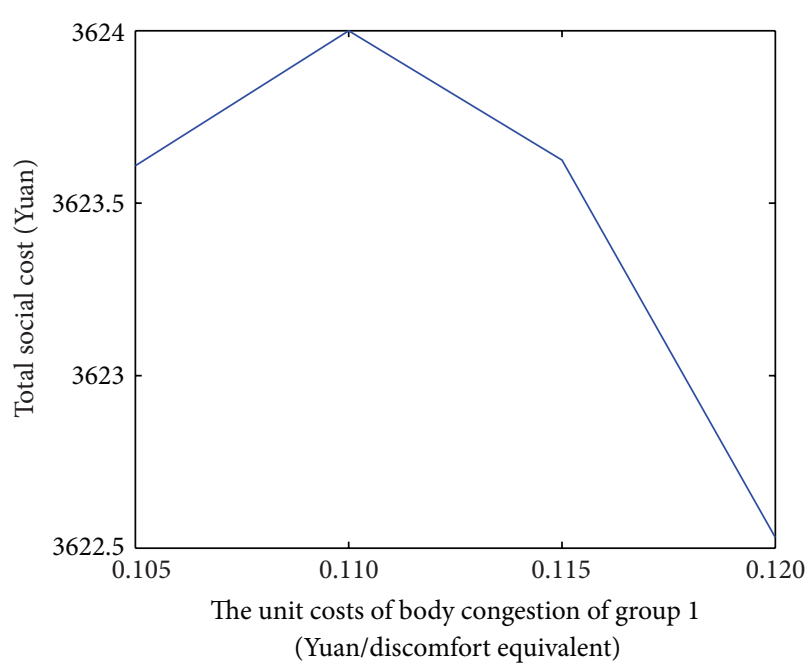

Figure 10: Total social cost versus $\theta_{1}$. 
improved and then decreases. The increase can be caused by the increase of the number of P\&R commuters; the decrease is due to the reduction of the queuing delay. This change implies that improving the service level of the subway in a certain range can reduce the total social cost.

\section{Conclusions}

The influence of parking fees on the mode choice and commuting behaviors in a competitive bottleneck transportation system with heterogeneous commuters was investigated in this article. It was found that nine equilibrium traffic patterns exist in the traffic system for different parking fees with the fixed traffic conditions. The necessary conditions for these equilibrium states are also given in this paper. Based on the most desired traffic pattern for traffic managers (both groups select both modes), we give the formulation of optimal parking fee. The findings in this paper have some implications to traffic management.

We intend to develop the present work in numerous directions. In particular, we are going to derive the values for the involved parameters on the basis of reliable data. Then we will include the total social welfare to be maximized with the elastic demand. Moreover, it could be interesting to perform analysis with respect to the time spent on searching for parking lots and spaces.

\section{Acknowledgments}

This work is financially supported by the State Key Laboratory of Rail Traffic Control and Safety (no. RCS2012ZT012), the National Basic Research Program of China (2012CB725400), the National Natural Science Foundation of China (nos. 71222101, 71071013, and 71131001), and the National High Technology Research and Development Program (no. 2011AA110303). The authors would like to thank the two anonymous referees for their helpful suggestions and corrections, which improved the content and composition substantially.

\section{References}

[1] J. Holguín-Veras, W. F. Yushimito, F. Aros-Vera, and J. (Jack) Reilly, "User rationality and optimal park-and-ride lot under potential demand maximization," Transportation Research Part $B$, vol. 46, no. 8, pp. 949-970, 2012.

[2] AASHTO, Guide for the Design of Park-and-Ride Facilities, American Association of State Highway and Transportation Officials, 1992.

[3] R. J. Spillar, Park-and-Ride Planning and Design Guidelines, Parsons Brinckerhoff, 1997.

[4] M. W. Horner and S. Groves, "Network flow-based strategies for identifying rail park-and-ride facility locations," SocioEconomic Planning Sciences, vol. 41, no. 3, pp. 255-268, 2007.

[5] AASHTO, Guide for Park-and-Ride Facilities, American Association of State Highway and Transportation Officials, 2004.

[6] B. Farhan and A. T. Murray, "Siting park-and-ride facilities using a multi-objective spatial optimization model," Computers and Operations Research, vol. 35, no. 2, pp. 445-456, 2008.
[7] J. Y. T. Wang, H. Yang, and R. Lindsey, "Locating and pricing park-and-ride facilities in a linear monocentric city with deterministic mode choice," Transportation Research Part B, vol. 38, no. 8, pp. 709-731, 2004.

[8] T. L. Liu, H. J. Huang, H. Yang, and X. Zhang, "Continuum modeling of park-and-ride services in a linear monocentric city with deterministic mode choice," Transportation Research Part $B$, vol. 43, no. 6, pp. 692-707, 2009.

[9] W. Vickrey, "Congestion theory and transport investment," The American Economic Review, vol. 59, no. 2, pp. 251-261, 1969.

[10] C. Hendrickson and G. Kocur, "Schedule delay and departure time decisions in a deterministic model," Transportation Science, vol. 15, no. 1, pp. 62-77, 1981.

[11] C. F. Daganzo, "The uniqueness of a time-dependent equilibrium distribution of arrivals at a single bottleneck," Transportation Science, vol. 19, no. 1, pp. 29-37, 1985.

[12] H. Yang and H. J. Huang, "Analysis of the time-varying pricing of a bottleneck with elastic demand using optimal control theory," Transportation Research Part B, vol. 31, no. 6, pp. 425440, 1997.

[13] Y. Cohen, "Commuter welfare under peak-period congestion tolls: who gains and who loses?" International Journal of Transport Economics, vol. 14, no. 3, pp. 238-266, 1987.

[14] R. Arnott, A. de Palma, and R. Lindsey, "The welfare effects of congestion tolls with heterogeneous commuters," Journal of Transport Economics and Policy, vol. 28, no. 2, pp. 139-161, 1994.

[15] G. Ramadurai, S. V. Ukkusuri, J. Zhao, and J. S. Pang, "Linear complementarity formulation for single bottleneck model with heterogeneous commuters," Transportation Research Part B, vol. 44, no. 2, pp. 193-214, 2010.

[16] Y. Liu and Y. Nie, "Morning commute problem considering route choice, user heterogeneity and alternative system optima," Transportation Research Part B, vol. 45, no. 4, pp. 619-642, 2011.

[17] Z. (Sean) Qian, F. (Evan) Xiao, and H. M. Zhang, "The economics of parking provision for the morning commute," Transportation Research Part A, vol. 45, no. 17, pp. 861-879, 2011.

[18] Z. Qian, F. Xiao, and H. M. Zhang, "Managing morning commute traffic with parking," Transportation Research Part B, vol. 46, no. 7, pp. 894-916, 2012.

[19] X. Zhang, H. J. Huang, and H. M. Zhang, "Integrated daily commuting patterns and optimal road tolls and parking fees in a linear city," Transportation Research Part B, vol. 42, no. 1, pp. 38-56, 2008.

[20] X. Zhang, H. Yang, and H. J. Huang, "Improving travel efficiency by parking permits distribution and trading," Transportation Research Part B, vol. 45, no. 7, pp. 1018-1034, 2011.

[21] H. J. Huang, "Fares and tolls in a competitive system with transit and highway: the case with two groups of commuters," Transportation Research Part E, vol. 36, no. 4, pp. 267-284, 2000.

[22] H. J. Huang, Q. Tian, H. Yang, and Z. Y. Gao, "Modal split and commuting pattern on a bottleneck-constrained highway," Transportation Research Part E, vol. 43, no. 5, pp. 578-590, 2007.

[23] V. van den Berg and E. T. Verhoef, "Congestion tolling in the bottleneck model with heterogeneous values of time," Transportation Research Part B, vol. 45, no. 1, pp. 60-78, 2011.

[24] T. Yao, T. L. Friesz, M. M. Wei, and Y. Yin, "Congestion derivatives for a traffic bottleneck," Transportation Research Part $B$, vol. 44, no. 10, pp. 1149-1165, 2010.

[25] F. Xiao, W. Shen, and H. M. Zhang, "The morning commute under flat toll and tactical waiting," Transportation Research Part B, vol. 46, no. 10, pp. 1346-1359, 2012. 


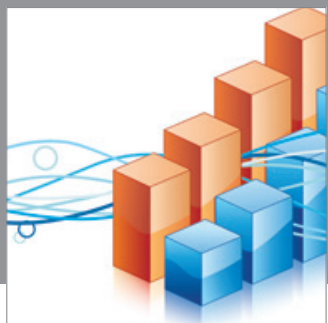

Advances in

Operations Research

mansans



The Scientific World Journal

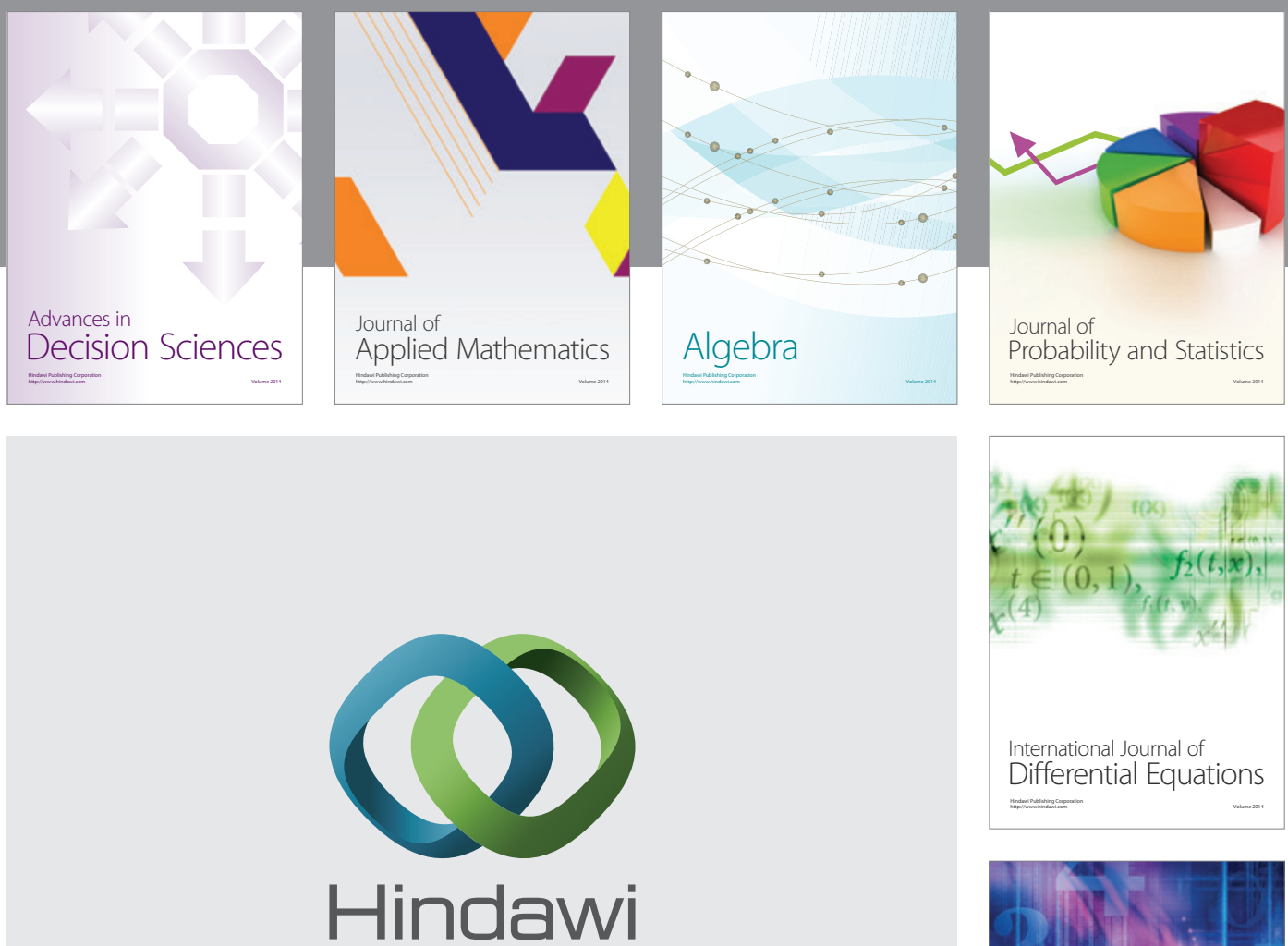

Submit your manuscripts at http://www.hindawi.com
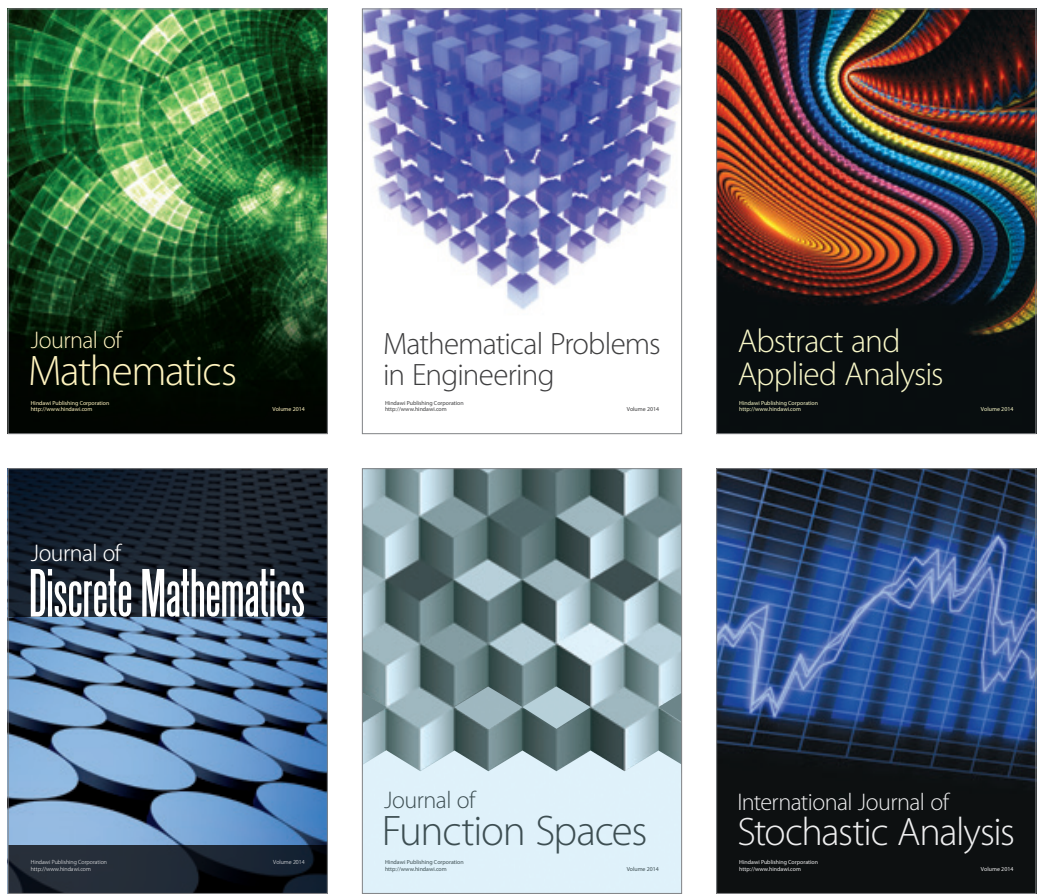

Journal of

Function Spaces

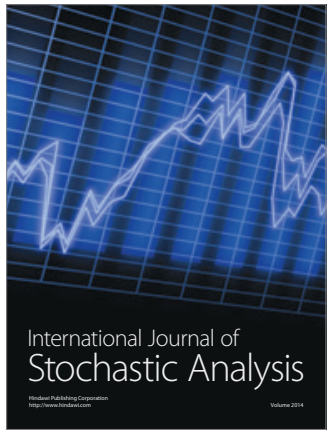

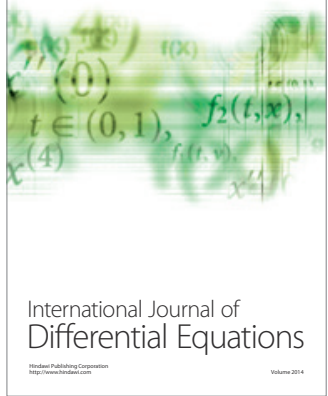
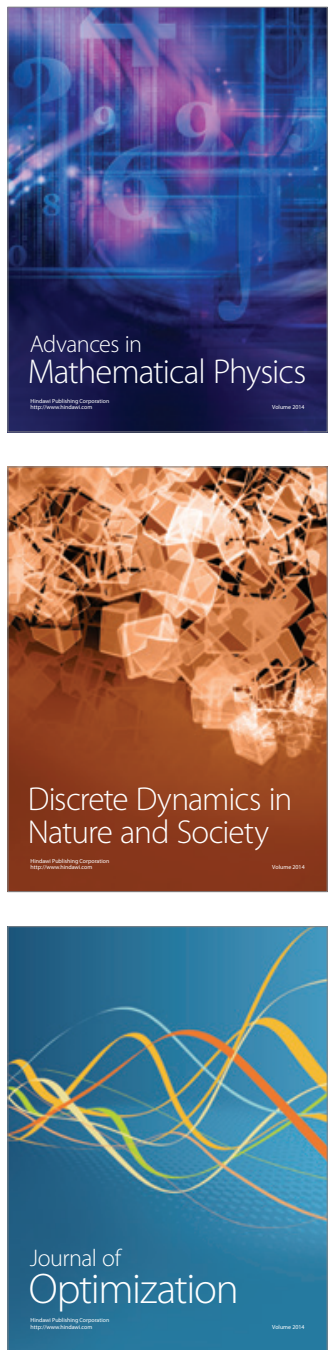